\title{
Die Morphologische Adaptation Deutscher Substantiva in Altweissrussischen Sprachdenkmälern aus der Sicht der Deutschen Historischen Morphologie
}

\author{
VON \\ HERMANN BIEDER
}

\section{Forschungsstand}

In den letzten Jahren wurden die dt. Lehnwörter in den awr. Sprachdenkmälern in zwei Dissertationen und einigen kleineren Beiträgen mehrmals behandelt. Den Beginn der Forschungsarbeiten $z u$ den älteren dt.-wr. Sprachbeziehungen bildete der lexikologischsprachgeschichtliche Artikel A. I. Zuuraŭskis über die Lexik dt. Herkunft im wr. Schrifttum des 15.-17. Jhs. ${ }^{1}$ Es folgten bald danach I. I. Cartkos Dissertation über die Lexik dt. Herkunft im Awr. ${ }^{2}$ und einige hiermit in Zusammenhang stehende kleinere Beiträge dieses Autors. ${ }^{3}$ Die phonetische und morphologische Adaptation diverser Lehnwortschichten im Awr., u. a. auch der Germanismen, hat auch A. M. Bułyka im Überblick dargestellt. ${ }^{4}$ Einen großen Teilbereich des dt. Lehnwortschatzes im Awr. hat O. M. Hałaj in ihrer soliden Dissertation bearbeitet.5 Die Autorin dieser neuesten und bisher besten einschlägigen Arbeit hat damit einen gewichtigen Beitrag zur Erforschung der älteren dt.-wr. lexikalischen Lehnbeziehungen geleistet. Von dieser wr. Germanistin stammen überdies auch noch einige Aufsätze zum dt. lexikalischen Einfluß auf die Sprache des awr. Schrifttums. ${ }^{6}$ Die bisher angeführten Autoren stützen ihre Arbeiten auf die lexikologische Analyse awr. Sprachdenkmäler des 14. bis 17. Jhs. Zum Unterschied von ihnen war I. M. Akułaŭ in seiner Dissertation über die Lehnwörter in der Struktur der wr. Sprache ${ }^{7}$ um eine Charakteristik aller Lehnwortschichten in der heutigen wr. Schriftsprache aus mehr allgemeinsprachwissenschaftlicher Sicht bemüht.

2. Morphologische Adaptation dt. Lehnwörter in anderen slav. Sprachen - Allgemeinsprachwissenschaftliche Problematik

Bevor wir mit der morphologischen Analyse der dt. Substantiva im awr. Schrifttum beginnen, empfiehlt es sich, die allgemeinlinguistischen Ergebnisse einiger neuerer Arbeiten zur morphologischen Adaptation von Lehnwörtern in den slav. Sprachen zusammen- 
zufassen. Diese Arbeiten bewegen sich gewöhnlich um folgende Fragestellungen: Nach welchen Prinzipien werden Lehnwörter morphologisch assimiliert? Worin zeigen sich Konvergenzen und Divergenzen der morphologischen Kategorien der Kontaktsprachen? Wie erfolgt die Substitution von Flexionselementen und können Flexionselemente entlehnt werden? Sind beim Lehnwort Verschiebungen der Grenze zwischen dem Grundmorphem (Stammorphem) und Flexionsmorphem möglich? Welche Genera und Deklinationsklassen der entlehnenden Sprache sind produktiv, bzw. nicht produktiv? Unter welchen morphologischen Voraussetzungen sind Lehnwörter deklinabel, bzw. nichtdeklinabel? Unter welchen Voraussetzungen werden Lehnwörter in Singularform als Pluralia tantum assimiliert?

Heute sieht man gewöhnlich jede Wortentlehnung als einen aktiven Prozeß: die entlehnende Sprache übernimmt das fremde Wort nicht passiv, sondern formt es um und paßt es den eigenen inneren Gesetzmäßigkeiten an. Das Lehnwort wird in das morphologische System der entlehnenden Sprache einbezogen indem es mit den entsprechenden grammatischen Kategorien versehen wird. ${ }^{8}$ An grammatischen Kategorien weisen sowohl die dt. als auch die slav. Substantiva Genus, Numerus und Kasus auf. Folglich kann man diesbezüglich von Gemeinsamkeiten der morphologischen Systeme sprechen. Ebenfalls gemein ist dem Dt. und den slav Sprachen die Dreigliєderung der Genuskategorie (Mask., Fem., Neutrum). Diese Einteilung geht im allgemeinen weder im Dt. noch im Slav. auf das natürliche Geschlecht zurück. W. Jung vermutet, daß sich das grammatische Genus des Dt. nach dem Klang der Endungen bestimmt hat." Während aber im Dt. schon seit langem der vermutliche frühere Zusammenhang zwischen Flexionsmorphem und Genus verwischt zu sein scheint, ist im Slav. die Korrelation dieser beiden unverkennbar. Während die dt. Substantiva sozusagen von vornherein ein bestimmtes grammatisches Genus haben und einige vage Regeln zur Bestimmung des Genus (besonders bei Substantiven mit gewissen Wortbildungsmorphemen) aufgestellt werden können, kann das grammatische Genus slav. Substantiva meistens unschwer am Wortauslaut abgelesen werden.

Nach K. Polański10 waren grammatisches Genus, Deklinationstyp und eventuell auch Numerus dt. Lehnwörter im Polab. abhängig

a) hauptsächlich von Faktoren formaler Natur (von der Form des nom. sg. des dt. Substantivs)

b) in einigen Fällen vom Einfluß eines heimischen Synonyms

c) in manchen Fällen vielleicht von der Tendenz, das dt. grammatische Genus zu bewahren, wodurch ein dt. fem. Substantiv auf Konsonanten in die slav. $a$-Stämme einbezogen werden konnte.

Die Polonistin I. Judycka ${ }^{11}$ vertrat die Überzeugung, daß auf das grammatische Genus dt. Lehnwörter in den poln. Dialekten der 
Masuren das dt. grammatische Genus Einfluß gehabt habe (d. h. unabhängig vom Wortauslaut).

Der Sorabist J. A. Sehnert kam zur Überzeugung, daß dt. Lehnwörter nach so regelmäßigen Mustern ins Osorb. entlehnt werden, $\mathrm{da} \beta$ es sogar möglich sein müßte, deren morphologische Integrationsergebnis vorherzusagen, wenn alle dt. dialektalen, phonologischen und morphologischen Kriterien berücksichtigt würden. ${ }^{12}$

Weniger optimistisch als Sehnert ist in dieser Hinsicht W. Doroszewski, der anhand von Lehnwörtern des heutigen Poln. nachweist, daß nicht immer nur der formal-strukturelle Faktor (die vokalische oder konsonantische Endung eines Lehnworts) das grammatische Genus im Poln. bestimmt, sondern daß auch sozio- und psycholinguistische Faktoren, wie der Grad der Kenntnis der Fremdsprache, Bildungsstand, soziale Herkunft des in den Sprachkontakt einbezogenen Individuums, mitentscheidend sein können. ${ }^{13}$ Nicht zuletzt von der soziolinguistischen Situation des Sprachkontakts hängt es ab, ob ein fremdes Flexionselement als selbständiges Morphem erkannt, vom Grundmorphem abgetrennt und durch ein eigenes Flexionselement substituiert wurde. Nur wenn die Analyse der morphematischen Struktur des Lehnworts mißlang, konnten fremde Flexionselemente in die entlehnende Sprache übernommen werden und zur Stammerweiterung führen. Das Grundmorphem des Lehnworts bleibt meistens morphologisch unverändert, seltener erfährt es eine Veränderung (Erweiterung) an der Grenze zu Flexions- oder Wortbildungsmorphemen. ${ }^{14}$ Aus der morphologischen Adaptation von Lehnwörtern ist ersichtlich, welche morphologische Kategorien zur Zeit der Entlehnung produktiv, bzw. nichtproduktiv waren. Lehnwörter werden ja normalerweise nur in produktive Kategorien der entlehnenden Sprache eingeordnet. Die Produktivität der Deklinationsklassen in den slav. Sprachen ist sicherlich nicht einheitlich. M. Martysjuk errechnete, daß bei der Genus-Assimilation dt. Lehnwörter im Russ. am produktivsten die Klasse der Mask. ist. Die Hauptmasse der Lehnwörter wird eben von dieser Klasse ausgedrückt $(87,2 \%)$. Nur ein eher kleiner Teil der Lehnwörter wurde in die Klasse der Fem. einbezogen $(12.93 \%$ ). Völlig passiv sei in dieser Hinsicht das Neutrum $(0,0 \overline{0} \%) .{ }^{15}$ Der hohe Anteil der Mask. ergibt sich daraus, daß die dt. Wortstämme meistens auf einen Konsonanten auslauten. Diese Genusverteilung dt. Lehnwörter im heutigen Russischen ist deswegen aufschlußreich, weil nach Martysjuk I. P. Mučnik für die moderne russische Schriftsprache insgesamt eine ganz andere Genusverteilung errechnet hat: 46\% Mask., 41\% Fem., $13 \%$ Neutra. ${ }^{16}$ Nach übereinstimmender Ansicht verschiedener Lexikologen ist die Klasse der Neutra in den einzelnen slav. Sprachen so gut wie nicht produktiv. Im Regelfall werden dt. Lehnwörter solchen slav. Deklinationsklassen zugeordnet, daß sie in allen Kasus flektiert werden können. In die Kategorie indeklinabler Wörter (unflektierbar in Kasus und Numerus, aber mit syntaktischer Kongruenz) werden $d t$. Lehnwörter nie eingeordnet, auch wenn sie eine für die entlehnende Sprache atypische Endung haben. In einem 
solchen Fall helfen sich die slav. Sprachen mit phonotaktischer Umformung des ungewöhnlichen Wortauslauts.

In semantischer Hinsicht ist das dt. Lehnwortmaterial in den slav. Sprachen nicht homogen. Meistens sind es Bezeichnungen für konkrete Gegenstände oder Personen, seltener abstrakte, stoffliche cder kollektive Substantiva. Aus diesem Grund ist die lexikalischgrammatische Kategorie des Numerus ungleichmäßig vertreten. Gewöhnlich werden dt. Substantiva in der Form des nom. sg. entlehnt und wie die slav. Erbwörter nach Numeri flektiert. Verhältnismäßig wenige werden nur im Singular oder nur im Plural verwendet.

Nach dieser Skizzierung der allgemeinsprachwissenschaftlichen Probleme, die bei der Untersuchung der morphologischen Adaptation dt. Lehnwörter in den slav. Sprachen auftreten können, soll auf die Ziele des vorliegenden Artikels eingegangen werden.

\section{Ziel der Untersuchung}

In den bisherigen Arbeiten wr. Lexikologen und Sprachhistoriker gründet sich die Charakterisierung der morphologischen Adaptation dt. Lehnwörter im Awr. gewöhnlich auf einen Vergleich des Wortauslauts und Genus awr. Germanismen mit der Auslautsstruktur und dem Genus der entsprechenden Lehwörter dt. Herkunft im Westslav. (vornehmlich im Poln., seltener im Cech.) und derjenigen dt. Etyma, die wahrscheinlich die Ausgangsformen bei den jeweiligen dt.-slav. Lehnbeziehungen bildeten. Bei dieser Gegenüberstellung dt. lexikalischer Einheiten und deren morphologischer Integrationsergebnisse in den slav. Sprachen werden erstere vorwiegend in mhd. normalisierter oder gar nhd. schriftsprachlicher Form angeführt. Viel zu wenig beachtet wird die starke dialektale Differenzierung des dt. Sprachraums und die chronologische Heterogenität des untersuchten lexikalischen Materials. Rekonstruktionsgrundlagen für dt.-slav. Lehnbeziehungen können aber nicht bloß überregionale Hochsprachen, wie sie das Mhd. und Nhd. darstellen, sondern müssen vor allem zeitgenössische regionale dt. Schreibsprachen (Kanzleisprachen) aus der dt.-slav. Kontaktzone sein, die doch bis zu einem gewissen Grad die historische Entwicklung dt. Dialekte widerspiegeln. Der lexikalische Einfluß der nhd. Standardsprache kann sich erst nach deren Formierung im 16. Jh. ausgewirkt haben. Außer Acht gelassen wurden bisher Fragen der historischen Morphologie des Dt., wie z .B. die Reduktion der Kasusflexion in der mhd. und besonders frühnhd. Periode und die damit verbundene Neuverteilung der morphologischen Kennzeichen des lexikalischen Materials auf Grund von grammatischer Analogie (Wechselwirkung verschiedener Flexionstypen, analoge morphologische Angleichungstendenzen und dgl.) $)^{17}$

Der vorliegende Beitrag setzt sich daher das Ziel, die morphologische Adaptation dt. Lehnwörter in den alten wr. Sprachdenkmälern und deren morphologische Assimilationsergebnisse im älteren zeitgenössischen Schrifttum anderer slav. Völker aus der Sicht der dt. 
historischen Dialektologie und Sprachgeschichte $\mathrm{zu}$ behandeln. Geklärt soll einerseits werden, welche Flexionsmorpheme dt. Lehnwörter im Awr. und in anderen slav. Sprachen sich als Substitution dt. Flexionsmorpheme deuten lassen, und andererseits soll gezeigt werden, welche Faktoren anderer Sprachebenen die Anpassung des fremden lexikalischen Materials an die morphologischen Systeme der slav. Sprachen zusätzlich beeinflussen konnten. Gemeint sind also nicht bloß Tendenzen auf der Ebene der eigentlichen Morphologie (Flexion), sondern auch Faktoren der Wortbildung, Lexikologie und Semantik sowie der Phonologie (Phonotaktik).

Behandelt werden nur dt. Substantiva mit formbildenden Flexionsmorphemen, dagegen werden Substantiva mit erkennbaren reihenbildenden Wortbildungsmorphemen (Suffixen, Präfixen), aber auch Nominalkomposita ausgesondert. Substantiva mit Wortbildungsmorphemen und Komposita (mit Flexions- oder Wortbildungselementen) berühren nämlich spezifische Fragen der historischen dt. und slav. Wortbildungslehre, sodaß diese besser in getrennten Aufsätzen behandelt werden.

Das lexikalische Material für die Untersuchung liefern dt. Lehnwörter aus awr. Sprachdenkmälern vom Anfang des 14. bis Ende des 17. Jhs. ${ }^{18} \mathrm{Da}$ ein Großteil der ins Awr. entlehnten Substantiva dt. Herkunft nicht unmittelbar aus dem Dt., sondern durch Vermittlung anderer slav. Sprachen entlehnt wurde (Entlehnung zweiter, dritter Stufe) muß die morphologische Adaptation der awr. Germanismen in den breiten Kontext analoger Adaptationsvorgänge der übrigen slav. Sprachen gestellt werden. Beobachtungen über Veränderungen und Schwankungen des Wortauslauts dt. Lehnwörter im Awr. wurden angestellt am Material historischer Wörterbücher des Dt. (Ahd., Mhd. und Mnd.) sowie an Wörterbüchern von dt. Dialekten, die ans westslav. Sprachgebiet grenzen (Bair., Osächs., Schles.). Weiters wurden für die Untersuchung zeitgenössische dt. kanzleisprachliche Texte aus dem dt.-poln. Grenzgebiet und Monographien zur dt. historischen Dialektologie ausgewertet.

Da die morphologische Adaptation dt. Substantiva im Slav. in erster Linie nach dem formalen Kriterium der Auslautstruktur erfolgt, ist es im Falle jedes einzelnen Lehnworts notwendig, sich über dessen sprachhistorische Entwicklung und dialektale morphologische Veränderung im klaren zu sein. Von der Klärung der Frage, ob der Auslaut des dt. Lehnworts zur Zeit und im Gebiet des dt.-slav. Sprachkontakts konsonantisch oder vokalisch war, hängt nämlich dessen Zuordnung zu einem bestimmten slav. Flexionstyp ab.

\section{Probleme der dt. historischen Morphologie}

Die Flexionsmorpheme des Dt. haben seit Beginn der sprachlichen Überlieferung bedeutsame Veränderungen erfahren. In der ältesten historischen Periode des Dt., im Ahd. und And., sind, wie im Germanischen, bei vielen starken (vokalischen) und schwachen (konsonantischen) Deklinationsklassen in unbetonter Stellung noch differenzierte, 
historisch auf verschiedene Stämme zurückgehende Flexionsmorpheme gegeben gewesen ${ }^{19}$ (ahd. part-a 'Barte, Axt', erb-i 'Erbe, Erbgut', balc-o 'Balken', sunt-e 'Sünde', sit-u 'Sitte'; han-o 'Hahn', hërz-a 'Herz', zung-a 'Zunge'). Aber bereits im Mhd. und Mnd. sind infolge des starken germanischen Akzents auf dem Grundmorphem sowie als Folge flexivischer und phonetischer Ausgleichstendenzen alle unbetonten Vokale zu einem gleichförmigen -e reduziert worden. ${ }^{20}$ (mhd. bart-e, erb-e, balk-e, sünt-e, sit-e; han-(e), hërz-(e), zung-e).

Eine weitere Auswirkung des germanischen Akzents ist die Apokope des auslautenden -e der Substantiva im Sg. und Pl. ("dem Haus" für “dem Hause”). Als apokopiert bezeichnet man alle Wortformen, die nach der normalen mhd. Grammatik ein Endungs-e haben sollen, dies aber verloren haben. Die Apokope ist in der Norm der nhd. Schriftsprache nicht durchgeführt. Die Apokope erfaßt in den heutigen Dialekten den Großteil des dt. Sprachgebiets, u. a. das Bair.Österr., Südthüring., südwestliche Obersächs. (= Oberdt. und südliche Ostmitteldt.), aber auch die ostnd. Kolonialmundarten, wie das Mecklenburg., Nordbrandenburg., Pommensche und Ostpreuß. (einschl. Hochpreußisch). Erhalten blieb auslautendes - $e$ dagegen im md. Gebiet, und zwar im Schles., Obersächs. (außer dem Südwesten), im Großteil des Thüring., im Nordhess. und Südbrandenburg. ${ }^{21}$ Die beiden nicht zusammenhängenden Gebiete im süd- und norddt. Raum sollen unabhängig voneinander entstanden sein. Die Apokope tritt zuerst im 12. Jh. in bair. schriftlichen Quellen auf. Im Nd. findet sich die Apokope in der zweiten Hälfte des 16. Jhs. zuerst in schriftlichen Quellen Mecklenburgs und breitet sich von da an aus. ${ }^{22}$ In der heutigen süddt., westdt. und norddt. Umgangssprache ist auslautendes $-e$ verbreitet abgeworfen. In der nhd. Standardsprache, die sich auf die omd. Sprech- und Schreibnorm stützt, ist auslautendes - $e$ in den meisten Fällen bewahrt (sogen. e-Saxonicum). Die omd. Dialekte, die den intensivsten Kontakt mit dem Poln. und durch dessen Vermittlung auch mit dem Awr. und Aukr. gehabt haben, bewahren noch weit konsequenter das auslautende $-e$ als die Schriftsprache.

Den ahd. und mhd. Substantiva mit vokalischem Morphem im nom. sg. stehen solche mit Nullmorphem im nom. sg. gegenüber (ahd. tag- $\varnothing$ 'Tag', gast- $\varnothing$ 'Gast', friunt- $\varnothing$ 'Freund', man- $\varnothing$ 'Mann'; mhd. tac- $\varnothing$ oder tag- $\varnothing$, gast- $\varnothing$, vriunt- $\varnothing$, man- $\varnothing$ ). Die Nullmorpheme dieser Deklinationsklassen finden unverändert ihre Fortsetzung im Nhd.

Aus diesen Ausführungen über die historische Entwicklung dt. Flexionsmorpheme läßt sich folgendes auf deren Substitution durch awr., bzw. überhaupt slav. Flexionselemente schließen: Von den dt. Substantiva mit Nullmorphem ist zu erwarten, daß sie bei der Entlehnung ins Awr. als endungslose Maskulina auftreten. Dt. Substantiva mit vokalischem Flexionsmorphem scheinen in der Regel als Feminina im Awr. auf. Wenn bei einem Lehnwort in allen Perioden dt. Sprachentwicklung und in allen Dialekten des dt. Sprachgebiets der Wortauslaut stabil geblieben ist, sind Genus-Schwankungen dt. Lehnwörter im Awr. und den übrigen slav. Sprachen weitgehend 
auszuschließen. Schwankungen beim grammatischen Genus der dt. Lehnwörter im Awr. sind vor allem aus der geschilderten dialektalen Differenzierung des dt. Wortauslauts zu erklären.

Das Vorhandensein von auslautendem -e, bzw. dessen Fehlen (Apokope) kann uns ferner die sprachgeographische Zuordnung eines Lehnworts zu einem bestimmten dt. Dialekt erleichtern. Nicht unterscheiden können wir dagegen mit diesem Kriterium dt.-slav. Sprachkontakte auf dialektaler und hochsprachlicher Ebene, weil sowohl die omd. Dialekte als auch die Norm der nhd. Schriftsprache das e-Saxonicum kennen.

Obwohl immer wieder über die Willkür der Genus-Verteilung im Dt. gesprochen wird, scheint doch ein gewisser Zusammenhang zwischen Flexionsmorphem und Genus zu bestehen. K. Polański ${ }^{23}$ hat daraufhingewiesen, daß die meisten mnd. Mask. auf einen Konsonanten endeten, also ein Nullflexionsmorphem aufwiesen und daß die zahlreichste Gruppe der mnd. Substantiva auf -e Fem. waren. Wegen des kons. Wortauslauts der Mask. und des Übergewichts der fem. Substantiva auf $-e$ im nom. sg. seien deshalb im ganzen dt. Sprachgebiet seit der mhd. und mnd. Periode die mask. Formen auf -e zurückgegangen. Wenn das mnd. fem. Substantiv im nom. sg. auf einen Konsonanten ende, komme es öfters zu einem Wechsel des grammatischen Genus vom Fem. zum Mask. oder Neutrum (nhd. Schnur f., aber mnd. snōr m.). Überhaupt zeige sich im Dt. eine deutliche Tendenz mit der Endung -e im nom. sg. das grammatische Genus der Fem. zu verbinden.

Ähnliche Beobachtungen lassen sich auch beim mhd. Wortschatz ansiellen: Mhd. Wörter mit Nullmorphem und $e$-Morphem haben entweder alternativ mask. oder fem. Genus (mhd. schram, schramme m./f. 'Schramme', dil, dille m./f. 'Diele') oder sie grenzen noch häufiger ihr Genus nach dem Flexionsmorphem ab: Mask. mit Nullmorphem und Fem. mit $e$-Morphem (mhd. valt $\mathrm{m}$. / valte f. 'Falte', mhd. brīsem m. / brīse f. 'Preise, Borte', mhd. krām m. / krām(e) f. 'Kram(ware, -laden)', mhd. kërp m. / kërbe f. 'Kerbe', mhd. slac m. / slage f. 'Schlag', mhd. spīl m. / spille f. 'Spille'). Von solchen mhd. lexikalischen Einheiten mit instabilem Wortauslaut sind Schwankungen zwischen Mask. und Fem. in den slav. Sprachen zu erwarten (wr. šram, ap. szram, poln. 16. Jh. szram und szrama, ukr. russ. šram; awr. falda, ap. fałd, poln. fałd und fałda; awr. kramı und krama, wr. krama, wr. dial. auch kram, poln. kram, čech. krám usw.). Diese Genusdifferenzierung kann im Mhd. mit einer semantischen Differenzierung verbunden sein (mhd. spil 'Spitze' / spille 'Spindel', slac 'Schlag (mit der Hand, mit einem Werkzeug oder einer Waffe)' / slage 'Werkzeug zum Schlagen, Hammer', schram 'Felsspalt, Loch' / schram(me) 'Schramme, lange Hauttoder Fleischwunde'. Natürlich haben auch solche semantische Differenzierungen sowie die Polysemie dt. Lexeme ihren Niederschlag im dt. Lehnwortschatz des Slav. finden mürsen (awr. šljaxъ, šljakъ 'Weg, Spur, Strecke, Bahn', awr. šljagъ 'Rand, Besatz, Borte', wr. šljax 'Weg', aber wr. šljaga 'Stampfe, Schlegel', ap. szlak 'Weg, Spur', älteres poln. szlak, szlag, ślak auch 
'Apoplexie; Gewandsaum; Taubenschlag; Kostenvoranschlag' aber szlaga 'Holzschlegel', in derselben Bedeutung ukr. šljaha). Die auf den omdt. Dialekten basierende nhd. Norm hat von den rivalisierenden Auslautformen den fem. auf $-e$ zum Sieg über die endungslosen mask. verholfen und diese letzteren zu Dialektformen werden lassen (mhd. valt, valte / nhd. Falte, mhd. dil, dille / nhd. Diele, mhd. kërp, kerbe / nhd. Kerbe, mhd. spīl, spille / nhd. Spille). Gelegentlich wurden auch mhd. endungslose Mask. und Neutra an die zahlreichen Fem. auf $-e$ im Nhd. angeglichen (spätmhd. brëm n. / nhd. Bräme, f., mhd. matraз m./n. / Matratze f.; vgl. awr. brama, materaç).

In der Folge werden die mit den morphologischen Kategorien Genus und Numerus zusammenhängenden Probleme der Adaptation dt. Lehnwörter im Awr. behandelt. Dabei werden wir uns auf die Korrelation der Flexionselemente dieser Lehnwörter im älteren Dt. und Awr. konzentrieren.

\section{Genus}

An Flexionsmorphemen weisen die untersuchten dt. Substantiva auf Nullmorphem (Typ Schacht-Ø), e-Morphem (Typ Schien-e), enMorphem (Typ Zeich-en). Das dt. Grundmorphem endet bei allen drej Typen auf einen Konsonanten.

\section{1 Substitution dt. Flexionsmorpheme durch awr. Flexionsmorpheme}

Fremde Flexionselemente werden bei der Entlehnung gewöhnlich als solche erkannt vom Grundmorphem abgetrennt und durch Flexionselemente der entlehnenden Sprache ersetzt. Die im Dt. zu beobachtende Tendenz, ein bestimmtes Flexionsmorphem mit einem bestimmten Genus zu verbinden und die im Slav. ohnehin gegebene Konvergenz von Flexionselement und Genus muß die Genus-Assimilation der dt. Lehnwörter im Awr. bedeutend erleichtert haben. Voraussetzung für eine bewußte, richtige Morphemanalyse dt. Lehnwörter durch Angehörige slav. Völker waren ausreichende Deutschkenntnisse, die sich entweder aus der dt.-slav. Lebenssymbiose in weiten Teilen Ostmitteleuropas und den daraus erwachsenen Formen dt.-slav. Bilinguismus ergeben hatten oder zumindest eine Folge de? weiten Verbreitung des Dt. als osteuropäischer Verkehrs- und Han delssprache waren.

\section{1. 1 Korrelationen des Wortauslauts dt. und awr. Substantiva}

\section{1. 1. 1 Dt. Nullmorphem durch awr. Nullmorphem substituiert}

Konsonantischer Auslaut des dt. Grundmorphems bewirkt, ohne Rücksicht auf das Genus des Lehnworts im Dt., die Eingliederung in die awr. Mask., die im nom. sg. ebenfalls durch Nullflexion gekennzeichnet sind. Wegen der fürs awr. phonologische System relevanten Opposition von Nichtpalatalität und Palatalität mußte das auslautende Konsonantenphonem des dt. Lehnworts als nichtpalatal, bzw. palatal strukturell eingeordnet werden. Ist der Wortauslaut als nicht- 
palatal integriert, wird das Lehnwort den Kontinuanten der mask. urslav. o-Stämme zugerechnet (awr. bunt's 'Bund, Aufstand' vojtъ 'Vogt', ginštъ 'Hengst, Streitroß', gaftъ 'Haft, Stickerei', daxъ 'Dach', druk` 'Druck, Buchdruck', žoldъ 'Sold', kgrafъ 'Graf', knextъ '(Kriegs) knecht', kunštı 'Kunst-, Gaukelstück', laštъ '(Wagen-, Schiffs)last', lios` 'Los, Schicksal', lioxъ 'Loch, Keller', lot`ъ 'Lot', maštъ 'Mast, Schiffsmast', ortъ 'Ort, Vierteltaler', pljacъ 'Platz', rimъ 'Reim' u. a.). Endet dagegen das Lehnwort auf einen palatalen Konsonanten, wird es meistens nach dem Muster der mask. jo-Stämme (selten der fem. i-Stämme) adaptiert (awr. palb, -ja 'Pfahl', calb, -ja 'Zoll (Maß)' sowie alle dt. Lehnwörter auf -el, wie awr. krukgel`̧ 'Krügel', rykgelb 'Riegel', pytelı '(Mchl)beutel', žakgel`s 'Segel', cvikelb 'Zwickel' u. a.). Die Palatalität des Wortauslauts ist allerdings graphisch nicht immer gekcnnzeichnet (awr. krukgel`ъ statt krukgels u. dgl.). Über die Eingliederung in die $i$-Stämme entscheidet weniger der palatale Wortauslaut, sondern eher die große Frequenz gewisser fem. awr. Substantiva auf -1' (solb, -i 'Salz', also celb, -i 'Ziel', palb, -i 'Pfahl').

Wichtigste Voraussetzung für ein stabiles mask. Genus des dt. Lehnworts im Awr. ist, daß der Wortauslaut im nom. sg. keinen chronologischen oder sprachgeographischen Modifikationen im Dt. untezworfen ist. Bei konstant stabilem Auslaut des dt. Lehnworts ist ein Genuswechsel von Mask. zu Fem. mehr oder minder auszuschließen. Tritt bei einem solchen Lehnwort neben dem zu erwartenden mask. Genus aber doch fem. Genus oder letzteres sogar ausschließlich auf, dann haben wir es mit ziemlicher Sicherheit mit einer Angleichung des Genus an jenes awr. Synonyma zu tun (awr. fasa ' $\mathrm{Faß}$ ' fem. wie awr. bočka 'dass.', wr. kaduška 'Kübel, Eimer', pasudzina 'Gefäß'; awr. turma 'Turm, Gefängnis' fem. wie awr. veža 'Turm'; awr. štaba '(Metall)stab, -stange' fem. wie wr. štanha 'Stange'; awr. šaxta '(Bergwerks)schacht' fem. wie wr. kapal'nja 'Bergwerk'). Eventuelle semantische Relationen mask. Lehnwörter zu mask. slav. Synonyma können sich natürlich nicht in Genusänderungen auswirken. Endet das Lehnwort im Dt. auf eine für das Awr. atypische Phonemverbindung, dann bewirkt das zwar keinen Genuswechsel oder Genusschwankungen, aber eine derartige Konsonantenfolge wird durch einen eingeschobenen Vokal silbisch neu strukturiert.

Wurde ein dt. Lehnwort in mehrere slav. Sprachen entlehnt, dann ist es natürlich in verschiedenartige semantische und morphologische Oppositionen hineingestellt, und folglich sind Genus-Divergenzen eines solchen Lehnworts in mehreren slav. Sprachen durchaus zu erwarten. $\mathrm{Da} \beta$ aber doch das Ergebnis der Genus-Adaptation eines dt. Lehnworts nicht in jeder slav. Sprache verschieden ist, ergibt sich wohl daraus, daß ein beträchtlicher Teil des Wortschatzes der slav. Sprachen gemeinslav. ist, also ähnliche semantische und morphologische Relationen herrschen müssen, und außerdem wurde ein derartiges Lehnwort häufig zunächst von einer bestimmten slav. Sprache morphologisch adaptiert und dann von ihr im Rahmen der innerslav. lexikalischen Lehnbeziehungen an andere slav. Sprachen morrhologisch unverändert tradiert. 
Die Opposition des mask. und fem. Genus kann bei ein und demselben dt. Lehnwort (das in diesem Fall meist verschiedener chronologischer und dialektaler Herkunft ist) in slav. Sprachen für semantische Differenzierung eingesetzt werden (awr. spižъ 'Erz, Glockenspeise' / spiža 'Mundvorrat, Proviant', wr. špic 'Spitze eines Bauwerks; Spitz (Hunderasse)' / špica 'Radspeiche; Bast zum Flechten von Bastschuhen', poln. rys 'Riß, Spalt' / rysa 'Merkmal', poln. luk 'Luke' / luka 'Lücke', poln. sztab 'milit. Stab' / sztaba 'Stab, Stange', poln. kaszt 'Gerüst im Bergwerksstollen' / kaszta 'Setzkasten' u. a.). Um verschiedene dt. Lehnwörter handelt es sich dagegen bei awr. cexъ 'Zeche, Innung' / cexa 'Zeichen', blexъ 'Bleiche' / blexa (Nebenform zu bljaxa) 'Blech'. Die dt. Lehnwörter fügen sich in die morphologischlexikalischen Systeme der slav. Sprachen ein, indem sie auch zu Einheiten des slav. Wortschatzes in morphologisch-lexikalische Oppositionen treten (awr. bramъ 'Verbrämung' / brama 'Tor', mordъ 'Pein, Qual; Mord' / morda 'Maul', ryzъ 'Ries (Papier), / ryza 'Ornat, Gewand', poln. spisa 'Spieß' / spis 'Verzeichnis', lina 'Leine' / lin 'Schleie', lista 'Liste' / list 'Brief', śluza 'Schleuse' / śluz 'Schleim').

\section{1. 1. 2 Dt.e-Morphem durch awr. a-Morphem substituiert}

Bei dt. Substantiva tritt das e-Morphem als formbildendes (genusbestimmendes) Element (dt. Rotte - awr. rota 'Rotte, Schar Soldaten'), aber auch als wortbildendes Ableitungssuffix auf. Im heutigen Dt. bildet das Suffix -e fem. Substantiva von verbaler und adjektivischer Basis. Die verbale Basis kann der Verbstamm des Infinitives (dt. Binde - awr. binda, dt. Schenke - awr. šinkъ 'Schenkrecht', dt. Rinne - awr. rinna) oder auch eine abgelautete Verbform (Präteritalstamm) (dt. fuhr, Fuhre - awr. fura) sein. Die Sachbezeichnungen können sich auf den Ort beziehen, an dem die betreffende Tätigkeit ausgeübt wird ${ }^{44}$ (dt. Bleiche - awr. blexъ). Zuweilen bezeichnet das $e$-Suffix auch Abstrakta (dt. Folge - awr. folbkga 'Folgeleistung; Erleichterung').

Das dt. formbildende und wortbildende $e$-Element hat eine Regularitätsentsprechung im awr. $a$-Morphem. Dt. Substantiva mit $e$-Morphem wurden bei mündlichem Sprachkontakt aus omd. Dialekten und bei schriftlichem Kontakt über die frühnhd. Schreibsprachen entlehnt. Wegen der geschilderten Apokopierung des e-Auslauts im Süden, Westen und Norden des dt. Sprachgebiets, die in häufigen Schwankungen des Wortauslauts (und seltener auch des Genus) in frühen dt. schreibsprachlichen Quellen ihren Ausdruck findet, kann die morphologische Adaptation eines derartigen Lehnworts im Awr. und den übrigen älteren slav. Schriftsprachen kein einheitliches Bild bieten. (awr. tarčъ ! tarča 'Tartsche, Schild'; apoln. fałd, poln. ab 17. Jh. fałd / fałda 'Falte'; awr. folbkga, apoln. folg / poln. 16. Jh. folga 'Folgeleistung; Erleichterung'; awr. skutъ, škotъ, älteres russ. škotъ, apoln. szkut / awr. škuta, apoln. szkuta 'Schute, Fluß- und Küstenschiff'; awr. stugъ / awr. stuga, apoln. stucha 'Kopftuch, Schleier, älteres dt. Stauche'). Eine Anzahl Lehnwörter mit mhd. normalem -e 
ist aber doch als stabiles awr. Fem. adaptiert worden (barva 'Farbe', buksa 'Buchse', busa 'Büse, Art Schiff', vanna 'Wanne', farba 'Farbe', varta 'Warte', vata 'Wate, Zugnetz', fara 'Pfarre', fukga 'Fuge', jupa 'Joppe', fljaša 'Flasche', mynca 'Münze', prasa 'Presse', kljampa 'Klampfe', kryga 'Kriege, Fischnetz'). Eine Reihe dt. Lehnwörter sind in awr. und apoln. Texten Fem., in ačech. oder čech. Denkmälern dagegen Mask.: Dieser Unterschied gibt deswegen zu denken, weil das Poln. und Cech. die betreffenden Lehnwörter aus verschiedenen dt. Dialekten entlehnt haben können (awr. folıkga, apoln. folga / ačech. folk 'Nachsicht'; awr. prasa, poln. prasa / čech. pres 'Presse'; apoln. figa, aber auch fig, fik / ačech. čech. fík 'Feige'; poln. fałda und fałd / čech. fald 'Falte' usw.). Während das čech. Sprachgebiet zum Großteil von apokopierenden dt. Mundarten (Bair.-Österr., Südthüring., südwestl. Obersächs.) umgeben ist, hatte das Poln. (und somit auch das Wr.) vorwiegend Kontakt mit den nichtapokopierenden dt. schles. Mundarten. Dieses Problem muß aber noch näher studiert werden. Tritt ein Lehnwort mit mhd. -e als Mask. auf, so kann dies, wie gesagt, entweder die Apokopie des dt. e-Morphems oder auch die Einwirkung des mask. Genus slav. sinnverwandter Wörter bedeuten. Eine Entscheidung dieser Frage kann nur mit Hilfe der Wortgeographie versucht werden.

Bei der Genus-Assimilation dt. Substantiva auf $-e$ wird auf slav. Boden nicht einfach dieses Element abgeworfen und ein Stamm auf einen Konsonanten gebildet, der den slav. Mask. eigen ist, wie sich dies M. Martysjuk vorstellt, ${ }^{25}$ sondern die slav. Völker wurden mit einem derartigen Lehnwort eben in apokopierter oder nichtapokopierter Gestalt vertraut. Diese beiden formalen Kriterien haben im Prinzip über mask. oder fem. Genus des dt. Lehnworts im Slav. entschieden.

Eine Reihe ursprünglich schwach flektierter dt. Substantiva auf $-e$ hat sich im Laufe der Zeit einer anderen Deklinationsklasse angeschlossen: Die schwache Flexionsform auf $-n$ drang in den nom. sg. und das Substantiv wurde dann in Analogie zu starken Substantiva auf -en (Typ Wagen) stark flektiert. ${ }^{26}$ Im heutigen Dt. ist zum Teil die Form mit -en üblich, - die $e$-Form wirkt dann gehobener, feierlicher -, zum Teil noch die e-Form. Derartige Substantiva wurden ins Awr. zum Teil als Fem. (awr. kasta '(Setz)kasten', mhd. kaste; 'šopa 'Schuppen, Stadel', mhd. schoppe; rama 'Rahmen', mhd. rame; kljuba 'Kloben', mhd. klobe; balıka 'Balken', mhd. balke; hierher gehört vermutlich auch awr. ryfa 'Reifen'), zum Teil als Mask. entlehnt (awr. gakı 'Haken', mhd. mnd. hāk(e); awr. knoţ 'Knoten, Docht, Lunte', mhd. knot(e); koç 'Kotze f., Kotzen m.', mnd. kotze, koz; boltъ 'Bolzen', mnd. bolt(e). Die Fem. reflektieren offensichlich den älteren Wortauslaut auf $-e$, die Mask. dagegen dürften auf apokopierten Wortauslaut zurückzuführen sein.

\section{1. 1. 3 Substitution von dt. ursprünglichem en-Morphem}

Neben einer Anzahl dt. Substantiva, bei denen ein Übergang von ursprünglichem -e $\mathrm{zu}-e n$ und Flexionswechsel durch einen flexi- 
vischen Angleichungsprozeß erfolgte, wurden drei Substantiva der starken Flexion mit ursprünglichem -en in awr. Denkmäler entlehnt, zwei davon als Fem. (awr. cexa 'Zeichen', ahd zeihan, mhd. zeichen; dakga 'Dolch', ahd. thegan, mhd. degan 'Degen', mnd. dagge 'Dolch'), eines als Mask. (awr. morkgъ 'Morgen (Flächenmaß)', ahd. morgan, mhd./mnd. morgen). Awr. dakga stammt wahrscheinlich aus mnd. degen. Wegen der wenigen Beispiele dieser Art lassen sich keine Regularitätsentsprechungen zwischen diesen dt. und awr. Flexionsendungen ablesen.

\section{1. 2 Erweiterung dt. einsilbiger Lehnwörter durch awr. fem. Strukturformanten}

Die Mehrzahl dt. Substantiva mit einsilbigem Grundmorphem wurde als einsilbige Mask. ins Awr. entlehnt (awr. blok's 'Block, Klotz', daxъ 'Dach', druk's 'Druck, Buchdruck' u. a.). Bei kurzen Lehnwörtern, besonders Einsilblern, läßt sich aber außerdem eine Tendenz zur Erweiterung des Grundmorphems durch ein slav. Suffix beobachten. Durch Derivation soll eine eventuelle weitere Reduktion des Einsilblers vermieden und die Identität kurzer Wörter bewahrt werden. Bei einer Reduktion liefe nämlich das Lehnwort Gefahr, seine Zeichenfunktion zu verlieren. Meistens werden durch diese Suffigierung potenzielle Mask. zu Fem.

Als solch rein formales Strukturelement tritt im Slav. in erster Linie das Suffix - $k$ - auf, das sich mit dem fem. Genus verbindet (awr. nyrka 'Niere', awr. mycka 'Mütze', awr. špilıka 'Spill(e)', awr. taška neben tašъ 'Tasche, Säckchen; Krambude', poln. mufka 'Muff', čech. flaška 'Flasche', russ. šponka 'Dübel' (eigentl. 'Span') u. dgl.). Da das Strukturmorphem $-k$ - mit dem diminutiv-hyperkorystischen Suffix $-k$ - homonym ist und außerdem nicht immer ein Sinnzusammenhang zwischen den Bedeutungen der beiden Morpheme ausgeschlossen werden kann, ist nicht immer klar, ob das Suffix - $k$ - nur einen Strukturformanten oder eine echte Diminuierung bezeichnet (z. B. russ. špulıka 'Spule (an Näh-, Webmaschinen u. ä.)'. In diesem Zusammenhang geht es nur um dt. Lehnwörter, die ohne das Suffix $-k$ - nicht überliefert sind. Allerdings könnte es sich auch einfach um eine Überlieferungslücke handeln, weil zu den fraglichen awr. Substantiven beispielsweise suffixlose Parallelen im älteren poln. Schrifttum zu finden sind (poln. myca, nyra, szpila). Allerdings sind diese poln. nichtsuffigierten Lehnwörter kaum gebräuchlich oder sie werden mit deutlich augmentativer Funktion (duża szpila) verwendet. Die Abgrenzung der Funktionen dieser homonymen Morpheme läßt sich nur dann durchführen, wenn ein ausreichendes lexikalisches Material zur Verfügung steht. Nachzuweisen wäre, daß solche suffixlosen Lehnwörter nicht existierten, bzw. die suffigierten nicht ausschließlich diminutiv verwendet werden. Jedenfalls ist eine gewisse Tendenz, den phonetischen Fremdkörper des dt. Lehnworts durch slav. Struktur- oder Wortbildungsformanten morphologisch und semantisch $\mathrm{zu}$ verdeutlichen, nicht zu übersehen. 


\section{2 Entlehnung des dt. Lehnworts samt Flexionsmorphem (Stammerweiterung)}

Bisweilen kann die Flexionsendung des fremden Worts bei der Entlehnung als Teil des Stammes empfunden werden. Die eigentliche Adaptation erfolgt, indem der erweiterte Stamm mit einem awr. Flexionselement versehen wird. Eine solche "verfehlte" Morphemanalyse erfolgte gelegentlich bei jenen Lehnwörten, welche die bereits angedeutete Verallgemeinerung der schwachen Flexion mitgemacht haben. Von diesem flexivischen Angleichungsprozeß wurden im Dt. Mask. und Fem. erfaßt.

\section{2. 1 Verallgemeinerung der schwachen Flexion bei den Mask.}

Im Dt., und zwar in der Schriftsprache wie auch in den Dialekten, ist, wie erwähnt, ein Teil der mask. Substantiva, der in der mhd. und mnd. Periode zur sogen. schwachen Deklination (Typ mhd. mnd. balke, gen. balken) gehört hatte, durch analogische Ausbreitung der Genitivendung -en auf den nom. sg. zur sogen. starken Deklination (nhd. Balken gen. Balkens) übergegangen. Dieser Teil der Mask., zu dem vorwiegend Bezeichnungen für Dinge gehören, ist somit mit den alten dt. Substantiva auf -en der starken Deklination zusammengefallen (Typ ahd. wagan, mhd. mnd. wagen, nhd. Wagen, gen. Wagens). ${ }^{2 \tau}$

Von dieser Gruppe dt. Mask. wurde nur ein einziges Lehnwort ins Awr. entlehnt, nämlich awr. balna f. 'Ballen': - $n$ - reflektiert den realisierten Flexionswechsel (und ist somit auch ein Kriterium für die Chronologie). Awr. kann nicht aus ahd. balo oder mhd. balle, sondern nur aus frühnhd. oder nhd. Ballen entlehnt sein. Ins übrige Slav. wurde dieses Lehnwort jedoch ohne -n-Element entlehnt (poln. 16. Jh. bella, balla, heutiges poln. bal, bala, bela; skr. bala). Awr. balna dürfte daher direkt aus dem Dt. entlehnt sein.

Vereinzelt wurden auch in andere slav. Sprachen dt. Lehnwörter mit diesem Flexionselement entlehnt. Der Stammauslaut von osorb. balkny n. pl. (neben osorb. balka, bolka) sowie von kärnt. sloven. polkna 'Balken' geht ebenfalls auf diese dt. Endung zurück (mhd./ mnd. balke m.). Auch dieses Lehnwort ist in die übrigen slav. Sprachen ohne den Formanten -en entlehnt worden (awr. balbka, wr. bèlıka, wr. dial. balıka, apoln. balk, balka, poln. $16 \mathrm{Jh}$. balka, belka, balk, bałk, bałek, poln. belka, russ. 15. Jh. balka, nsorb. balka, bałka). Ein Reflex dieser morphologischen Angleichung ist in ačech. kaštna kaštna neben kašta und in skr. kašna zu erkennen (vgl. mhd. kaste, mnd. kaste, kast, kass, aber nhd. Kasten). Awr. kasta 'Setzkasten', neueres poln. kaszta neben kaszt, sloven. košt, kašt verraten dagegen, daß dt. -en als Flexionselement aufgefaßt und daher weggelassen wurde.

\section{2. 2 Verallgemeinerung der schwachen Flexion bei dem Fem.}

Während die Verallgemeinerung der schwachen Flexion bei einem Teil der Mask. schon in der frühnhd. Periode alle dt. Dialekte erfaßte 
und diese Entwicklung zur hochsprachlichen Norm erhoben wurde, ist der analoge Angleichungsprozeß bei den Fem. auf die bair.-österr. Dialekte beschränkt geblieben²8 (z. B. niederösterr. lautn 'Laute', fletn "Flöte”, kœ:n "Kelle”, biksn 'Büchse', brukn 'Brücke'). Weder in den frühnhd. Schreibsprachen noch in der nhd. Schriftsprache hat sich diese morphologische Angleichung durchsetzen können.

Fast alle awr. fem. Lehnwörter dt. Herkunft haben einen nichtpalatalen Stammauslaut (Typ vag-a 'Waage'), nur drei Lehnwörter, die Fem. im Dt. sind, nämlich awr. ljutnja 'Laute', fletnja 'Flöte' und kelınja 'Kelle' weisen auffallenderweise palatalen Stammauslaut auf (ljutn'-, fletn'-, kel'n'-) und sind im Vergleich zum dt. Grundmorphem um das Flexionselement erweitert. Es drängt sich die Frage auf, ob das $n$-Element als Reflex eines dt. dialektalen morphologischen Merkmals zu erklären ist oder ob wir es einfach mit dem slav. struktuellen Formanten $-n^{\prime}(a)$ zu tun haben.

Für A. Brückner ${ }^{29}$ jedenfalls wurde einfach älteres dt. lüte durch poln. -nia erweitert. J. Łośs0 und St. Kochman ${ }^{31}$ dachten bei poln. lutnia auch an eine Angleichung an den poln. Formanten -nia. Im Poln. sind Wörter auf -nia eine semantisch ganz uneinheitliche Gruppe (jutrznia 'Morgengottesdienst', ćwiertnia 'Viertel (einer Einheit)', pochodnia 'Fackel', wiśnia 'Weichselkirsche', trześnia ('wilde) Kirsche'. Sie sind durchwegs Reliktbildungen ohne Produktivität. Von dem sporadischen poln. Morphem -nia mit allgemein strukturaler Funktion sind natürlich die häufigen slav. homonymen Wortbildungsmorpheme $-n^{\prime}(a)$ zu unterscheiden, die gewöhnlich Nomina loci, deprekative Nomina actionis und Kollektiva mit pejorativer Schattierung bezeichnen. Im älteren Poln., Wr. und Ukr. wurden zahlreiche Nomina loci sowie Nomina officinae auf $-n^{\prime} a$ von dt. Lehnwörtern motiviert (poln. krochmalnia 'Stärkerei; Stärkefabrik', masztalnia 'Marstall', cegielnia 'Ziegelei', szmelcarnia 'Schmelzerei'). Aber nur in den wenigsten Fällen ist poln. -nia bei dt. Lehnwörtern nur formbildend und nicht wortbildend aufgetreten, und was noch bedeutsamer ist, nur bei jenen Mask. und Fem., die im Dt. den geschilderten flexivischen Angleichungsprozeß mitgemacht haben (poln. sztolnia, russ. štolınja '(Bergwerks)stollen', mhd. stolle m., aber niederösterr. dial. štojn; poln. luśnia 'Leuchse f., Kippstange beim Ochsenwagen'; poln. putnia 'Bütte, Butte f.' niederösterr. butn 'Holzeimer' mhd. büite, bütten 'Gefäß, Bütte') Cech. kuchyně, poln. kuchnia, ostslav. kuxnja 'Küche' reflektieren ebenfalls eine alte dt. Wortform auf - $n$ - (ahd. chuhhina, mhd. küchen, kuchīn, frühnd. kuchin < lat. coquina), nur daß -en als Flexionsendung der schwachen Deklination aufgefaßt und der nom. sg. 'Küche' neu gebildet wurde. Wie ersichtlich, enden heute die nhd. Mask. auf -en (Stollen), bei den nhd. Fem. dagegen hat sich $-n$ nicht durchgesetzt (Bütte, Leuchse), bzw. sie haben dieses Element in Umkehrung dieses Prozesses sogar verlieren müssen (Küche, weiters Ferse, Kette u. a.) ${ }^{32}$ Daraus ergibt sich, daß das slav. formbildende Element $-n^{\prime} a$ durch das dt. Flexionselement bedingt sein muß. Allerdings ist es durchaus 
denkbar, daß sich diese morphologischen Bildungen am Auslautsmodell slav. Erbwörter oder anderer früher Lehnwörter zusätzlich orientiert haben (poln. fletnia, lutnia wie ćwiertnia; luśnia wie wiśnia, trześnia; kielnia wie patelnia 'Pfanne'; sztolnia, kuchnia wie sypialnia 'Schlafzimmer'). Ein Zusammenwirken beider morphologischer Faktoren, also eine Verschmelzung des dt. Flexionsformanten mit dem slav. Strukturformanten, erscheint als die wahrscheinlichste Lösung des Problems.

Die morphologische Adaptation der erwähnten drei Lehnwörter gibt Anlaß zum Nachdenken, weil diese Nomina instrumenti doch einen phonotaktisch problemlosen Wortauslaut hatten und Formen wie *l'uta, *kela, *flet(a) ohne weiteres denkbar gewesen wären. Und tatsächlich sind diese Lehnwörter in den Denkmälern der anderen slav. Sprachen mit - $n$ oder ohne dieses vertreten.

Im Poln. ist seit Mitte 15. Jh. nur lutnia gebräuchlich, und ebenso tritt im Cech. dieses Lehnwort nur mit Stammerweiterung auf (ačech. lútna, čech. loutna). In poln. Gestalt ist das Lehnwort ins Wr., Ukr. und Russ. gelangt. Osorb. Denkmäler schwanken zwischen lutnja, lutna und luta, lawta. Weiters findet sich skr. schriftsprachliches lutnia, aber skr. umgangssprachliches oder dial. lauta f., laut m.; slvk. schriftsprachl. lutna, slvk. dial. lavta. Formen mit $-n$ - sind die entschieden älteren, solche ohne - $n$ - späte Entlehnungen aus dem Nhd.

In poln. Texten ist vom 16. Jh. bis in unsere Tage ein Schwanken zwischen fletnia und flet festzustellen. Im 16. Jh. überwiegt fletnia, heute dagegen gilt fletnia als veraltet, durchgesetzt hat sich flet. Alten Kontakt mit dem Bair.-Österr. müssen čech. fletna und skr. dial. fletna reflektieren. Auf md., bzw. nd. Herkunft oder auch schriftsprachliche Entlehnung deuten osorb. fleta, flěta, nsorb. flejta, älteres russ. flejt, fleta, heutiges russ. wr. bulg. flejta, weil sie das -n- Element nicht reflektieren. In der schriftlichen Überlieferung des Dt. wird immer - $n$ gemieden (mhd. vloite, floit, flöute, mnd. floite, jidd. fleit).

Neben häufigerem awr. kelınja findet sich auch awr. kelja, kellja. Im heutigen Wr. und Russ. kelınja und kelıma. Russ. kelınja seit 17. bezeugt. In apoln. Texten (15. Jh.) kiella, killa, aber schon im 16. Jh. kielnia neben kiela, kiele, kiella, ab 17. Jh. nur mehr kielnia gebräuchlich. Cech. kelna, aber osorb. khela, slvn. kela, čela. Dt. Ưberlieferung: mhd. mnd. kelle.

An den Belegen läßt sich eine Fernwirkung der bair.-österr. Dialekte übers Cech. aufs Poln. und Ostslav. und übers Slvn. aufs Skr. ablesen.

\section{3. Awr. Genera und Deklinationsklassen}

5. 3. 1 Awr. Genera

Das Dt. und Wr. besitzen drei Genera. Das Genus des Etymons im Dt. spielt für die Genus-Assimilation im Awr. aus den erwähnten formal-strukturalen Gründen kaum eine Rolle. Nichtderivierte dt. Lehnwörter wurden mit awr. Flexionsmorphemen oder auch kombi- 
niert mit awr. Strukturformanten und Flexionsmorphemen in die Genus-Kategorie eingegliedert.

Mask.: Flexionsmorphem $-\varnothing$ nach hartem Stammauslaut (Typ drot- $\varnothing$ ) und weichem Stammauslaut (Typ calb-Ø)

Fem.: Flexionsmorpheme - $a$ (Typ bind-a), $-\varnothing$ nach weichem Stammauslaut gelegentlich (Typ stalb-Ø), Allomorpheme -ovb- $\varnothing$, -evь- $\varnothing$, -va (Typ panovb, panevь, panva);

Strukturformanten $-n^{\prime}-,-k$ - kombiniert mit Flexionsmorphem $-a$ (Typen fletnja, mycka).

Neutr.: Flexionsmorphem -o (Typ clo).

Auf gewisse Konvergenzen zwischen Wortauslaut und Genus im Dt. und Wr. wurde bereits hingewiesen. Konsonantischer dt. Wortauslaut wurde (von wenigen Ausnahmen des Typs stalb 'Stahl' abgesehen) mit den ähnlich auslautenden awr. Mask. assoziiert. Ebenso problemlos wurde eine morphologische Korrelation zwischen dem dt. $e$-Morphem und dem $a$-Morphem der awr.Fem. hergestellt. Im ganzen Untersuchungszeitraum ist das mask. und fem. Genus uneingeschränkt produktiv gewesen. Das dt. e-Element (Typ Fuhre) wurde bezeichnenderweise nie mit den awr. Neutra auf -e- (Typ mor'e) assoziiert. Folgende Gründe mögen hiefür maßgebend gewesen sein: Die awr. Flexionsendung $-e$, obligatorisch nach Palatalen, ist in morphematischer Hinsicht bloß als Allomorphem des Morphems -o anzusehen. In phonologischer Hinsicht konnte wegen der unterschiedlichen Strukturierung des dt. und wr. phonologischen Systems zwischen dem nichtpalatalen auslautenden Konsonanten des Typs Fuhre und dem palatalen Auslautskonsonanten des Typs mor'e auch keine Korrelation hergestellt werden. Schließlich scheinen die awr. Neutra auf -o nur in der frühesten Zeit dt.-slav. Sprachbeziehungen in geringem Maß produktiv gewesen zu sein. Für diese Annahme spricht die geringe Zahl und das hohe Alter der awr. Neutra dt. Herkunft.

Awr. clo n. 'Zoll (Abgabe)' und calb m. 'Zoll (Maß)', beide aus demselben $\mathrm{dt}$. Zoll, aber zu verschiedenen Zeiten entlehnt, veranschaulichen die Veränderungen in der Produktivität slav. Neutra. Awr. clo (in apoln. und ačech. Denkmälern schon im 14. Jh. belegt) ist wesentlich älterer Herkunft als awr. calb, da im Dt. erst Anfang 16. Jh. Zoll als Längenmaß in Gebrauch kommt und hierauf ziemlich rasch in die west- und ostslav. Sprachen dringt.

Die übrigen awr. Neutra dt. Herkunft sind Adaptationen dt. isolierter Bildungen auf $-e r$ und $-e l$, bei denen die Herstellung der Motivationsbeziehung und die Feststellung der Derivationsbasis Schwierigkeiten bereitet (awr. olstro 'Pistolenhalfter' - dt. Holster; awr. rudło; awr. koldro neben koldra 'Bettdecke' - dt. Kolter; awr. pudlo 'Schachtel' - dt. Pudel, Paudel). Vgl. noch russ. dyšlo 'Deichsel', aber poln. dyszel, poln. 16. Jh. auch dyszla; ${ }^{33}$ weiters poln. lustro 'Spiegel; Kronleuchter (veralt.)' aus dt. Lüster, Luster. Polysemantisches dt. Futter wurde ins Poln. zweimal, und zwar im 
als Neutr. und in der neueren Zeit als Mask, mit verschiedener Semantik entlehnt (apoln. futro 'Pelz; Unterfutter', aber poln. umgangssprachl. futer 'Nahrung, Essen'. Für diese Neutra auf -ro, -lo standen zahlreiche slav. Neutrabildungen Modell (vgl. poln. Derivate auf -dło, wie bydło 'Vieh', zwierciadło 'Spiegel', prawidło 'Regel', Powidło; poln. piętro 'Stockwerk', Adverbia wie poln. jutro 'morgen', chytro 'schlau'; russ. sedlo 'Sattel', russ. Adverbia bystro 'rasch', pestro 'bunt' u. a.). Čech. povidla np. pl., gen. pl. povidel wurde nach dem Muster dt. -el- Bildungen ins Dt. entlehnt. Auf die morphologische Adaptation dt. Wortbildungssuffixe kann aber in diesem Zusammenhang nicht weiter eingegangen werden.

\section{3. 2 Awr. Deklinationsklassen}

Sehen wir von Reliktwörtern diverser altertümlicher Deklinationsklassen ab, so finden wir sowohl im Awr. wie auch im modernen Wr. drei grundlegende Deklinationsklassen, die alle eine Fortsetzung urslav. Formensysteme darstellen: o/jo-Stämme (Mask. u. Neutra),

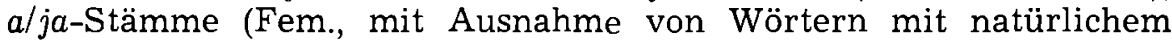
mask. Genus), $i$-Stämme (Fem.).

5. 3. 2. 1 Nach den awr. o/jo- Stämmen werden Mask. (Typen blikь 'metallische Verzierung (eigentl. Blick)'; cals 'Zoll (Maß)' und Neutra (Typ futro 'Pelz') flektiert.

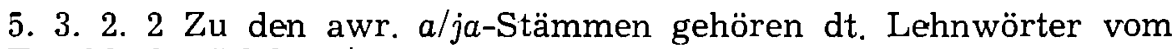
Typ kljuba 'Kloben'/ljutnja 'Laute'.

Ein auffallendes Phänomen ist die Angleichung einiger dt. Lehnwörter an die Reste der urslav. $\bar{u}$-Deklination (Typ kry, -ъve 'Blut'), deren Model sich schon zur Zeit der ältesten slav. Sprachdenkmäler im Auflösungszustand befunden hat. Im Slav. sind die Wörter dieser Deklination zum Teil in den $\bar{a}$-Stämmen (bulg. cărkva 'Kirche'), zum Teil in den $i$-Stämmen (wr. ljuboŭ, -bvi 'Liébe') aufgegangen, wobei sie einen sekundär aus den obliquen Kasus entwickelten Nominativ angenommen haben. Schon zu Beginn unseres Jahrtausends begannen Lehnwörter im Slav. dieses Modell zu meiden. Konservativer und widerstandsfähiger war dieses Modell nur im Westslav., in den südwestlichen Dialekten des Ukr., im Slvn. und in einigen westlichen Dialekten des Skr. Schneller verfiel es dagegen in den ostslav. Sprachen, in den meisten skr. Dialekten und im Bulg.-Maked. ${ }^{34}$

Die in den awr. Sprachdenkmälern enthaltenen Germanismen mit morphologischen Merkmalen der urslav. $\bar{u}$-Deklination lassen sich chronologisch in zwei Gruppen teilen: Entlehnungen aus dem Ahd., vor der einzelsprachlichen Differenzierung des Ostslav. (vor 14. Jh.) die zustandekamen (awr. panva/panovb/panevb 'Pfanne'; konva/konovb/ konovs 'Kanne'; skalva 'Waagschale') und Entlehnungen aus dem Mhd. oder Frühnhd., die schon in einer Periode eigenständiger wr. Sprachentwicklung erfolgten (awr. rynva 'Rinne', lištva 'Leiste', latva 'Latte'). Die Kontinuanten von urslav. *pany, -sve 'Pfanne' haben gesamtslav. Verbreitung erfahren, jene von urslav. *kony, -ъve 'Kanne' sind zumindest im ganzen West- und Ostslav. gegeben. Für 
aruss. skaly, awr. skalva 'Waagschale' finden sich nur spärliche Belege in frühen ostslav. Urkunden, die im Handelskontakt mit Livland entstanden. Der anlautenden sk-Verbindung nach zu schließen wurde skalva aus dem livländ. Mnd. oder gar aus dem Skand. entlehnt. In jüngerer Form wurde dieses Lehnwort ein zweites Mal ins Awr. als šalja 'Waagschale', bezeichnenderweise als ja-Stamm, entlehnt. In manchen awr. Texten vom Ende des 16. Jh. findet sich opanova, oponova 'Pfanne', mit prothetischen o-, eine Erscheinung, die in heutigem wr. dial. apanoŭka wiederkehrt. Die ungewöhnliche Endung von awr. paneva, opanova, opaneva läßt sich entweder als Kreuzung von Flexionselementen der $a$ - und $i$-Deklination oder auch als mißlungene Wiederherstellung der Ableitungsbasis aus den Diminutiven panovka, panevka 'kleine Pfanne' deuten. Awr. panovъ, konovъ zeigt den ostlav. Reflex des alten Halbvokals ъ, awr. panevъ, konevs dagegen den westslav. (poln.). Häufiges -ovъ statt -ovb reflektiert die Depalatalisierung der Labiale im Awr. Diese älteren Lehnwörter sind im Awr. mit Formen aus dem Paradigma der $a$-Deklination und $i$-Deklination vertreten. Die jüngeren dt. Lehnwörter, die einen Reflex der $\bar{u}$-Stämme aufzuweisen scheinen, nämlich awr. rynva neben ryna, latva neben lata, lištva neben lišta haben keine Wortformen nach dem Modell der $i$-Stämme ausgebildet (*rynovs, *latovb, *lištovs nicht belegt, aber poln. lisztwa/lisztew 'Leiste').

Handelt es sich bei diesen spätmittelalterlichen oder frühneuzeitlichen Lehnwörtern aus dem Dt. um eine echte Reaktivierung der $\bar{u}$-Deklination oder haben auch andere Faktoren eine Rolle gespielt? Die Wiederbelebung eines zum Stillstand gekommenen morphologischen Typs ließe sich erwarten, wenn ein Wort dieses Typs besonders prominent wird und sich ihm deswegen andere Wörter anschließen. Dies läßt sich von awr. rynva annehmen, das sich an das Modell des weitverbreiteten und gutbelegten awr. konva angeglichen haben dürfte. Dagegen haben sich awr. lištva, latva ohne Rücksicht auf die Semantik mit Sicherheit den gängigen nomina actionis deverbativer Herkunft auf -tva (Typ wr. brytva 'Rasiermesser', malitva 'Gebet', bitva 'Schlacht') angeschlossen. Zwar haben diese Derivate keine direkte Beziehung $\mathrm{zu}$ den $\bar{u}$-Stämmen, da sie mit Hilfe des Suffixes -tva gebildet sind, doch haben auch sie im Slav. die Einwirkung der Modelle auf $-y$, - $v$ ve erfahren (poln. brzytwa und brzytew, čech. břitva und břitev 'Rasiermesser'). Das Suffix -tva diente als Modell für die Adaptierung einer Reihe anderer dt. Lehwörter im Slav. (wr. ukr. russ. dratva, poln. dratwa, dratew, čech. dratev, slvk. dratva, nsorb. drětwa, osorb. gratej, gen -wje '(Schuster)draht'; osorb. nsorb. ratwa 'Ratte'; nsorb. watwa 'Watte').

Die an die Formanten der $\bar{u}$-Deklination, bzw, an das -tva-Suffix angepaßten d.t. Lehnwörter erfuhren eine Veränderung ihrer Morphemstruktur durch die Verschiebung der Grenze zwischen Wurzel und Formanten infolge sekundärer Zergliederung des Worts in morphologische Teile, die nicht mit dem ursprünglichen etymologisch motivierten Stand übereinstimmen (sogen. Perintegration), ${ }^{\mathbf{3 5}}$ z. B. wr. lištv-a, konaŭ-k-a im Vergleich zu dt. Leist-e, Kann-e. 
Von den rivalisierenden awr. Formen lištva/lišta, latva/lata, rynva/ ryna haben sich im heutigen Wr. und Ukr. beide Typen behauptet (wr. lištva, ukr. lyštva, ukr. rynva, aber wr. lata, ryna, ukr. lata).

5. 3. 2. 3 Die awr. $i$-Deklination ist im Vergleich zur o-Deklination und $\bar{a}$-Deklination bei dt. Lehnwörtern kaum produktiv: Für eine Angleichung an die $i$-Stämme kommen nur jene dt. Lehnwörter in Frage, die einen palatalen Auslaut haben. Das einzige dt. Phonem, das im Rahmen der awr. Phonemoppositionen als palatal eingereiht wurde, war der Sonant $/ I^{\prime} \%$. Lehnwörter mit auslautendem $-l$, wie Pfahl, Stahl, Ziel, Zoll, Diel(e), waren in die Konkurrenz der wenig gängigen $i$-Stämme und der vitaleren jo-Stämme hineingestellt. Durchgesetzt haben sich gewöhnlich die gebräuchlicheren jo-Stämme, dagegen lassen sich nur wenige Wortformen der $i$-Deklination finden: awr. celь 'Ziel' und palı 'Pfahl' sind gewöhnlich mask. jo-Stämme, nur selten flektieren sie wie fem. $i$-Stämme; für awr. cals 'Zoll (Längenmaß)' und dylı 'Diele, Brett' lassen sich überhaupt nur Wortformen der jo-Stämme finden. Nicht selten allerdings, etwa im Fall von awr. stalb 'Stahl', läßt sich die Zugehörigkeit einer Wortform $\mathrm{zu}$ dem einen oder anderen Paradigma wegen Homonymität von Kasusendungen oder mangels syntaktischer Kongruenz nicht bestimmen. Im heutigen Wr. sind diese Lehnwörter durchwegs Fem., zum Teil nach der $i$-Deklination (cèlb, -i, stalb, -i), zum Teil nach der $j a-$ Deklination (dylja, -i, palja, -i, calja, -i) flektiert. Dieselben Lehnwörter gehören im heutigen schriftsprachlichen Ukr. in der Mehrzahl denselben Flexionsklassen an (ukr. cilb, -i, stalb, -i, palja, -i, aber calb, -ja), dagegen heben sie sich in dieser Hinsicht deutlich vom modernen hochsprachlichen Poln. ab (cel, $-\mathrm{u}$, pal, -a, dyl, -a, cal, -a, aber stal, -i). Die morphologische Instabilität der genannten Lehnwörter im poln. und ukr. Schrifttum des 18.-19. Jh. (älteres poln. pala, -i neben pal, -u, cala, -i neben cal, -a; ukr. palja, -i neben palb, -ja) sowie in den heutigen wr. und russ. Dialekten (wr. dial. pal; russ. dial. palja neben pal) läßt in diesen Sprachen auf langwährende Wechselwirkungen zwischen den einzelnen Flexionsklassen oder auch unterschiedliche Synonymenbeziehungen schließen.

\section{Numerus}

Das semantische Feld der Numerusfunktion sind bekanntlich nur zähl- oder berechenbare Appellativa. Voraussetzung der Zählbarkeit ist die Gegliedertheit sowie Gleichartigkeit der Elemente einer Gegenstandsklasse. Von den mehreren hundert Lehnwörtern dt. Herkunft im awr. Schrifttum erfüllen weitaus die meisten diese Voraussetzungen, soda $\beta$ sie problemlos in die awr. grammatischsemantische Singular-Plural-Opposition einbezogen werden konnten (awr. drots 'Draht' - droty, barta 'Barte' - barty, futro 'Pelz' futra usw.). Nur wenige nichtzählbare Stoffbezeichnungen (awr. ircha 'Sämischleder', krejda 'Kreide', cynъ 'Zinn', krachmalı 'Stärke, eigentl. Kraftmehl', kopervasъ 'Kupferwasser') und Abstrakta (awr. 
goldъ 'Huldigung', musъ 'Muß, Zwang', kglanecъ 'Glanz', brakъ 'Ausnahme, eigentl. Brack') haben keine korrelativen Numerusformen.

Manche dt. Lehnwörter sind in awr. Texten Pluraliatantum, also Substantiva mit lexikalisierter Pluralform (awr. obcukgi 'Hufzange', pljudry 'Pluderhose', pukli 'runde erhabene Metallverzierung von Bucheinbänden, Gürteln, Waffen und Zaumzeug (eigentl. Buckel)' cukgli 'Zügel', šrakgi 'Schragen, Gestell', bukšteli 'Bogengestell') oder sie werden zumindest nur selten im Singular, gewöhnlich im Plural verwendet (awr. lejç 'Leitseil', gewöhnlich pl. lejcy). Diese Lehnwörter sind auch im Poln. Plt. oder im Sg. kaum gebräuchlich (poln. obcęgi, pukle, cugle, szragi, buksztele, pludry). In den Plt. ist der alte grammatische Inhalt zwar noch spürbar, im Grunde aber schon verblaßt, er spielt keine aktuelle Rolle mehr. Wir können erahnen, daß awr. pljudry (Bezeichnungen für 'Hose' sind in vielen europ. Sprachen Plt., wie russ. brjuki, štany, poln. spodnie, engl. trousers, frz. pantalons) sowie awr. obcukgi (wie russ. nožnicy 'Schere', poln. nożyce 'dass.', poln. kleszcze 'Zange', alle plt.) wegen der Zweiheit dieser Gegenstände, aber auch awr.' bukšteli und šrakgi (wie auch fachsprachl. skr. šarage plt.) wegen einer Vielzahl (Zwei-, Vierheit) der Bauelemente als Plt. aufgefaßt werden. Selbst im Fall von awr. cukgli 'Zügel' und lejcy 'Leitseil' dürfte die Assoziation mit der Zweiheit dieser Gegenstände (zwei Längen) den Ausschlag für das Plt. gegeben haben. Die Pluralform von lejcy könnte auch phonetisch zu erklären sein: In gewissen dt. Dialekten konnte auslautendes $-l$ vokalisiert werden (z. B. österr. leitsæu 'Leitseil') und der sich ergebende reduzierte Auslaut als slav. Pluralelement identifiziert worden sein. Awr. pukli verdankt seinen Pluralgebrauch wohl der Vorstellung von einer Vielzahl gleichartiger kleiner Gegenstände.

\section{Zur Phonotaktik und Wortbildung}

Dt. Lehnwörter haben zwar mitunter einen Wortauslaut mit für das Awr. atypischen Konsonantenverbindungen, aber trotzdem wurden sie deshalb nie in die Kategorie indeklinabler Wörter eingereiht. Vielmehr wurden solche fremde unaussprechbare Konsonantenverbindungen im nom sg. in der Regel durch einen in den obliquen Kasus beweglichen Vokal aufgelöst und auf diese Weise formal an ein awr. Wortbildungselement angeglichen. Geht dt. auslautendem $-z$ ein Konsonant (gewöhnlich Sonant oder Nasal) voraus, so wird diese Phonemverbindung an das awr. Suffix $-e c$ angeglichen (dt. -rz > awr. -rесъ, dt. $-\mathrm{lz}>$ awr. -lecъ, dt. $-\mathrm{nz}>$ awr. -nесъ: dt März > awr. marecъ, dt. Schmalz > awr. šmalecъ, dt. Tanz > awr. tanecъ). Setzt sich die dt. auslautende Phonemverbindung aus einem Konsonanten (Nasal, Sonant) sowie den Verschlußlauten $k$ oder g ( $=$ auslautverhärtet [k]) zusammen, so führt dies zur formalen Angleichung an das awr. Suffix -okъ (dt. -nk $>$ awr. -nokъ, dt. -lk $>$ awr. -l-okъ, dt. -rk > awr. -r-okъ, dt. -ng [-nk] > awr. -n-okъ: dt. Trunk, Schank, Schrank(e) $>$ awr. trunokъ, šanokъ, šranokъ; mhd. marschalc ; awr. 
maršalokъ; dt. Jahrmark(t), Vorwerk > awr. jarmarokъ, fol'varokъ; dt. Gang, Ring > awr. kganokъ, rinokъ). Diese formale Angleichung des Wortauslauts dt. Lehnwörter an die äußere Gestalt awr. Suffixe ist ein Beweis für die Assimilationskraft der alten wr. Schriftsprache. Nur weniger gängige Lehnwörter, wie awr. šancъ 'Glücksfall, Chance', švankъ 'Schaden, Beschädigung, eigentl. Schwank(en)', šinkъ '(Aus)schenken, Schank von Getränken', haben keine Anpassung des phonotaktisch fremden Wortauslauts an awr. Wortbildungselemente erfahren.

In funktionaler Hinsicht konnte ein derartiges scheinbares Suffix selbstverständlich nicht als echtes Wortbildungsmorphem fungieren, da es sich hierbei nicht um ein Verhältnis von Basis und Derivat handelte. Awr. maršalokъ 'Marschall' macht zwar den Eindruck eines echten Derivats, ${ }^{\mathbf{3 6}}$ weil wir es mit wr. maršal 'dass.', einer viel späteren neuerlichen Entlehnung aus dem Dt. oder Frz. in Verbindung bringen können, aber in Wirklichkeit ist dieses mhd. Lehnwort ein Kompositum aus mhd. marc 'Streitroß' und schalc 'Knecht', das die eben geschilderte morphologische Umgestaltung erfuhr. Dt. Lehnwörter auf -есъ und -okъ flektieren wie awr. Wörter mit echten Suffixen, d. h. mit beweglichen Vokalen (mareç, gen. marca, maršalokъ, gen. maršalka). Die angeführten Lehnwörter haben eine suffixähnliche Umgestaltung ihres Auslauts auch im älteren Poln. erfahren, wobei im allgemeinen Regularitätsentsprechungen zwischen poln. $-e c$, $-e k$ und awr. $-e c\urcorner,-o k v$ festzustellen sind (poln. taniec, ganek - awr. tanecъ, kganokъ).

\section{8) Folgerungen}

Die morphologische Adaptation von Lehnwörtern berührt nicht nur die eigentliche Morphologie (Flexion), sondern auch andere sprachliche Ebenen, wie Lexik und Semantik, Wortbildung sowie Phonologie (Phonotaktik).

\section{A N M ER K U N G E N}

1. A. I. Zuraŭski, 'Leksika njameckaha pachodžańnia ŭ biełaruskaj piśmiennaści XVXVII stst.', Biełaruskaja mova. Minsk, 1965, S. 60-73.

2. I. I. Cartko, Leksika nemeckogo proischoždenija $v$ starobelorusskom jazyke, Avtoref. kand. diss., Minsk, 1967, 24 S.

3. Cartko, 'Hiermanizmy narodnaj biełaruskaj movy i ich adpaviedniki u niamieckaj movie', Biełaruski linhvistyčny zbornik, Minsk, 1966, S. 146-53;

'Praniknieńnie hiermanizmaŭ ŭ leksiku starabiełaruskaj movy', Vieści AN BSSR, 1 , Sier. hram. navuk, Minsk, 1966, S. 120-7.

4. A. M. Bułyka 'Fanietyčnaja i marfalahiěnaja adaptacyja zapazyčańniaŭ ŭ starabiełaruskaj movie', Vieści AN BSSR, 6, Sler, hram. navuk, Minsk, 1970, S. 115-24.

5. O. M. Hałaj, Bytovaja leksika nemeckogo proischozdenija v starobelorusskom jazyke na materiale pis'mennych pamjatnikov XV-XVII vv., Avtoref. kand. diss., Minsk, $1977,23 \mathrm{~S}$.

6. Halaj, 'O morfologiceskom osvojenii zaimstvovannych slov v starobelorusskom jazyke', Voprosy obščego $i$ slavjanskogo jazykoznanija, Dnepropetrovsk, 1976, S. 105-8.

'Da siemantyčnaha asvajeńnia niamieckich zapazyčańnlaŭ u starablełaruskaf movie', Vieśnik BDU, sier. 4, 2, Minsk, S. 34-8.

'Słovaŭtvaralnaja zdolnaść hiermanizmaŭ u starabiełaruskaj movie', Biełaruskaja mova i movoznaŭstva, 4, Minsk, S. 80-8.

'Fanietyčnaje varjiravańnie słoŭ niamieckaha pachodžańnia ŭ starabiełaruskaj movie,' Vieśnik BDU, sier. 4, 2, Minsk, 1976, S. 31-5. 
7. I. M. Akułaŭ, Zaimstvovanija $v$ strukture belorusskogo jazyka, Avtoref. kand. diss., Minsk, 1977, 19 S.;

'Inšamoŭnaja leksika ŭ sučasnaj biełaruskaj movie', Biełaruskaja mova $i$ movaznaŭstva, II, Minsk, 1974, S. 10-15.

8. Näheres über Lehnwörter aus allgemeinsprachwissenschaftlicher Sicht bei Ju. $S$. Maslov, Vvedenije $v$ jazykoznanije, Moskva, 1975, S. 256-67.

9. W. Jung, Grammatik der deutschen Sprache, 5. Aufl., Leipzig, 1973, S. 264.

10. K. Polański, 'Zasady morfologicznej adaptacji niemieckich rzeczowników zapożyczonych w języku polabskim', Zeszyty naukowe UJ, 60, Prace językoznawcze, 5, Kraków, 1963, S. 40.

11. Judycka, 'Typy zapożyczeń niemieckich $\mathrm{w}$ gwarach Warmii i Mazur', Poradnik językowy, Warszawa, 1954, zesz. 8, S. 8.

12. J. A. Sehnert, 'The morphology of German loanwords in Upper Sorbian', Beiträge zur sorbischen Sprachwissenschaft, Bautzen, 1968, S. 150.

13. W. Doroszewski, 'U rodzaju gramatycznym wyrazów obcych w języku polskim', Studia i szkice językoznawcze, Warszawa, 1962, S. 275.

14. Vg1. I. T. Jacenko, 'Assimiljacija zaimstvovannych imen susčestvitel'nych na urovne formal'no-gramaticeskich pokazatelej', Issledovanija po gramatike i leksikologii, Kiev, 1966, S. 94.

15. M. Martysjuk 'Rodovaja assimiljacija nemeckich zaimstvovanij v russkom Jazyke', Studia Rossica Posnaniensia, Poznañ, 1970, 1, S. 181.

16. ibid., S. 175 .

17. V. M. Zirmunskij, Deutsche Mundartkunde, Berlin, 1962, S. 414.

18. Der Zugang zur Kartei des Wörterbuchs der awr. Sprache wurde mir bei einem Studienaufenthalt in Minsk am Institut für Sprachwissenschaft der Weissiuss. Akad. der Wissenschaften, Abteilung für Geschichte der wr. Sprache, von Prof. Dr. A. I. Zuraŭski dankenswerterweise ermöglicht.

19. St. Sonderegger, Althochdeutsche Sprache und Literatur, Berlin-New York, 1974, S. 172-7.

20. Zirmunskij, op. cit., S. 411-4, 159.

21. ibid., S. 159-61.

22. W. König, dtv-Atlas zur deutschen Sprache, München, 1978, S. 159.

23. Polański, op. cit. (Anm. 10), S. 37-40.

24. W. Fleischer, Wortbildung der deutschen Gegenwartssprache, 4. Aufl., Tübingen, 1975,

25. Martysjuk, op. cit. (Anm. 15), s. 180 .

26. W. Jung, op. cit., S. 288 .

27. Zirmunskij, op. cit., S. $415,420$.

28. $i$ bid., S. 431.

29. A. Brückner, Stownik etymologiczny języka polskiego, Wyd. III, Warszawa, 1974, S. 304.

30. J. Łoś, Grammatyka polska, II, Lwów-Warszawa-Kraków, 1925, S. 33.

31. St. Kochman, Polonica w leksykografii rosyjskiej XVII wieku, Warszawa-Wrocław, 1975, S. 55.

32. F. Kluge, Etymologisches Wörterbuch der deutschen Sprache, 20. Aufl., Berlin, 1967, S. 409.

33. Vgl. M. Karpluk, 'Wahania rodzaju gramatycznego zapożyczeń pod wplywem wyrazów bliskoznacznych na przykładzie pol. chomąto, dyszel', PAN - Oddział w Krakowie, Prace Komisji jezykoznawstwa, 37 Studia indoeuropejskie, Wrocław-Warszawa, 1974, S. 87-90.

34. S. B. Bernštejn, oŁerk sravnitel'noj grammatiki slavjanskich jazykov: Ceredovanija. Imennyje osnovy, Moskva, 1974, S. 240.

35. Z. Klemensiewicz, T. Lehr-Spławiński, S. Urbańczyk, Gramatyka historyczna języka polskiego, Wyd. III, Warszawa, 1965, S. 172-3.

36. Elne solche Vorstellung hat von diesem Lehnwort anscheinend L. L. Humec'ka, 'Morfematyðni substytuciji v najdavnisych ukrajins'kych zapozyčennjach z pol's'koji movy', Pytannja slov'jans'koho movoznavstva, 9, L'viv, 1963, S. 52. 